\title{
Observational cross-sectional study to evaluate the effects of self- medication with topical agents used by patients for superficial fungal skin infection at tertiary care hospital in Mumbai
}

\author{
Sunanda A. Mahajan', Surbhi S. Deshmukh², Jatin R. Rawal ${ }^{2 *}$
}

\begin{abstract}
${ }^{1}$ Department of Dermatology, Seth G. S. Medical College and KEM Hospital, Parel, Mumbai, Maharashtra, India ${ }^{2}$ Department of Infectious Diseases, KEM Hospital, MUHS, Mumbai, Maharashtra, India
\end{abstract}

Received: 03 March 2020

Revised: 09 April 2020

Accepted: 13 April 2020

*Correspondence:

Mr. Jatin R. Rawal,

Email: jatin008rawal@gmail.com

Copyright: (C) the author(s), publisher and licensee Medip Academy. This is an open-access article distributed under the terms of the Creative Commons Attribution Non-Commercial License, which permits unrestricted non-commercial use, distribution, and reproduction in any medium, provided the original work is properly cited.

\begin{abstract}
Background: Superficial fungal infections of the hair, skin, and nails are a major cause of morbidity in the world and their incidence continues to increase. While self-medication is also one of the major health concerns for health authorities worldwide. The aim of the study was to evaluate the effects of topical agents used by patients for selfmedication, to enlisting the topical agents used by patients for self-medication, to ensure by whose suggestion's patients engaged in self-medication.

Methods: It was an observational cross-sectional study which was conducted at the outpatient department (OPD) of dermatology in tertiary care hospital. A detailed history of the participant was recorded regarding topical agents used for self-medication and their effects were documented in a predesigned case record form.

Results: The population consisted of 128 males and 87 females. All these 215 participants had used 61 topical agents of different brand names and the majority of were irritant, corticosteroids and antifungal much high in use. Among 215 participants, 111 had temporary relief from the infection and reoccurred after stopping its use. 98 showed aggravation in their infection among which 90 showed effects like irritation, burning and itching at the application site, while 8 participants showed dryness or flaking of skin.

Conclusions: Self-medication is widely practiced among patients with a superficial fungal skin infection in Mumbai. A pharmacist was the major source for obtaining these topical agents. We can conclude, there is a need to counsel and discourage patients from self-medication and regulations for dispensing the prescribed drugs should be made stringent.
\end{abstract}

Keywords: Fixed dose combinations, Irritant, Steroids, Self-medication, Superficial fungal infection

\section{INTRODUCTION}

Humans are exceptional among vertebrates, wherein their living tissue is directly exposed to the outside world. ${ }^{1}$ Worldwide in general practice, $2 \%$ of consultations account for dermatological conditions. ${ }^{2}$ The pattern of skin disease varies from one country to another and across different parts within the same country. ${ }^{3}$ In developing countries like India, skin disease is prevalent due to ignorance. ${ }^{4}$ The prevalence of skin disease in developing countries is higher due to improper sanitation, low hygiene and high rate of person to person contact. ${ }^{5,6}$

Treatment is the most important part of both curing the disease as well as in preventing the spread of communicable diseases. ${ }^{7}$ Many dermatological diseases 
require a longer time of treatment and thus, the impact on the physical, social, psychological and financial burden on the patient. The quality of life with these distressing diseases is also reduced due to appearance. ${ }^{3}$

The practice of self-medication is common in India and many other parts of the world, especially in economically deprived communities. ${ }^{8}$ The urge of self-care, feeling of sympathy towards family members in sickness, lack of health services, poverty, ignorance, misbelieves, extensive advertisement and availability of drugs in other than drug shops are responsible for growing trend of selfmedication. ${ }^{9}$ It has been reported that drug use is influenced by the socioeconomic and demographic characteristics of consumers. ${ }^{10}$ Consequently, in developing countries, pharmaceutical outlets often serve as the first contact point of healthcare. In addition, lax medical regulation has resulted in the proliferation of counterfeit drugs that are in high demand for the treatment of highly prevalent diseases. ${ }^{11}$ FDC containing topical steroids and irritant like salicylic acid are one of this which are being often used for various skin lesions and also being used often by patients as over the counter medications (OTC). ${ }^{12}$

Identifying the predictors of self-medication, the data will provide meaningful evidence based on which intermediations can be planned at the level of the patient, physician and institute which will help to formulate targeted strategies to discourage unnecessary selfmedication, thereby preventing the occurrence of drugrelated problems associated with it. Keeping these facts in contemplation, the present study was planned in the inoutpatient department of dermatology.

\section{METHODS}

It was a hospital-based; observational cross-sectional study which was conducted in the dermatology outpatient department (OPD) at a Seth G. S. Medical College and K. E.M Hospital, Parel, Mumbai between the periods of February 2018 to June 2018. Prior approval from the institutional ethical committee was obtained before initiation of the study. After obtaining informed consent, 360 patients were screened for the study among which 215 patients with superficial fungal infection of 18 years and above ages and both sexes were recruited. To maintain confidentiality right of the enrolled participant, subject ID were allotted to each enrolled participant. Patients who were unable to respond to verbal questions, vulnerable population such as children's, pregnant women, patients with psychological disease history, etc. and those unwilling for participation were excluded.

A predesigned case record form eliciting clinicodemographic variables, characteristics of topical medication use, the source of prescription, and their effects were administered to all enrolled participants. Patients were then examined by a single observer to determine the pattern of presentation and adverse effects of medications used. Additional signs and symptoms of skin diseases were noted. The data were analysed using descriptive statistics.

\section{RESULTS}

Our study population consisted of $128(60 \%)$ male and 87 (40\%) female. The mean age of the study population was 32.6 years, ranging from 18 to 77 years. The mean duration of dermatophytosis in our study population was 2.5 months, and $46.97 \%$ of the cases had the disease ranging from 1 to 3 months, $35.81 \%$ ranging from 4 to 12 months and $17.22 \%$ ranging from 13 to 36 months duration.

Clinical examination of the study population revealed tinea cruris as the most common presentation $(50.23 \%)$, followed by tinea corporis $(23.25 \%)$, tinea pedis $(4.65 \%)$, and an overlap of these was (20\%) (Table 1).

Table 1: Clinico-epidemiological profile of the study population.

\begin{tabular}{|ll|}
\hline Clinico-epidemiological variables & N \\
\hline Age (years) & \\
\hline $18-27$ & 101 \\
\hline $28-37$ & 48 \\
\hline $38-47$ & 36 \\
\hline $48-57$ & 22 \\
\hline $58-67$ & 7 \\
\hline $68-77$ & 1 \\
\hline Educational qualification & \\
\hline Below secondary & 79 \\
\hline Secondary & 50 \\
\hline Higher secondary & 42 \\
\hline Under graduate & 10 \\
\hline Graduate & 33 \\
\hline Post graduate & 1 \\
\hline Duration of disease & \\
\hline Less than 01 month & 18 \\
\hline 1 to 3 months & 101 \\
\hline 4 to 6 months & 34 \\
\hline 7 to 9 months & 12 \\
\hline 10 to 12 months & 31 \\
\hline 24 months & 17 \\
\hline 36 months & 2 \\
\hline Clinical presentation & 108 \\
\hline Tinea cruris & 50 \\
\hline Tinea corporis & 4 \\
\hline Tinea faciei & 43 \\
\hline Tinea pedis & \\
\hline Overlap & \\
\hline & \\
\hline
\end{tabular}

Surprisingly, only $7.45 \%$ of the study population consulted a dermatologist on developing a rash, whereas the majority $(92.55 \%)$ used OTC topical medications recommended by pharmacists and friends/relatives, 
general practitioners, and television advertisement (Table 2).

Table 2: Source of over-the-counter medication prescription.

\begin{tabular}{|ll|}
\hline Source & $\mathbf{N}(\%)$ \\
\hline Advertisement & $19(8.84)$ \\
\hline Family members and relatives & $32(14.88)$ \\
\hline Friends and neighbours & $26(12.09)$ \\
\hline General practitioner & $16(7.44)$ \\
\hline Pharmacist & $98(45.58)$ \\
\hline Self & $8(3.72)$ \\
\hline
\end{tabular}

After investigating the participants it came to know that, 215 participants have used 61 different topical agents by Brand Name, which was categorized on the basis of nature of its active ingredients to different types like antifungal, antibacterial, antiseptic, antiparasitic, combinations (steroids- antifungal, steroids- antibacterial and steroids - antifungal - antibacterial ), corticosteroids, hydroxynathrone and irritant (Figure 1).

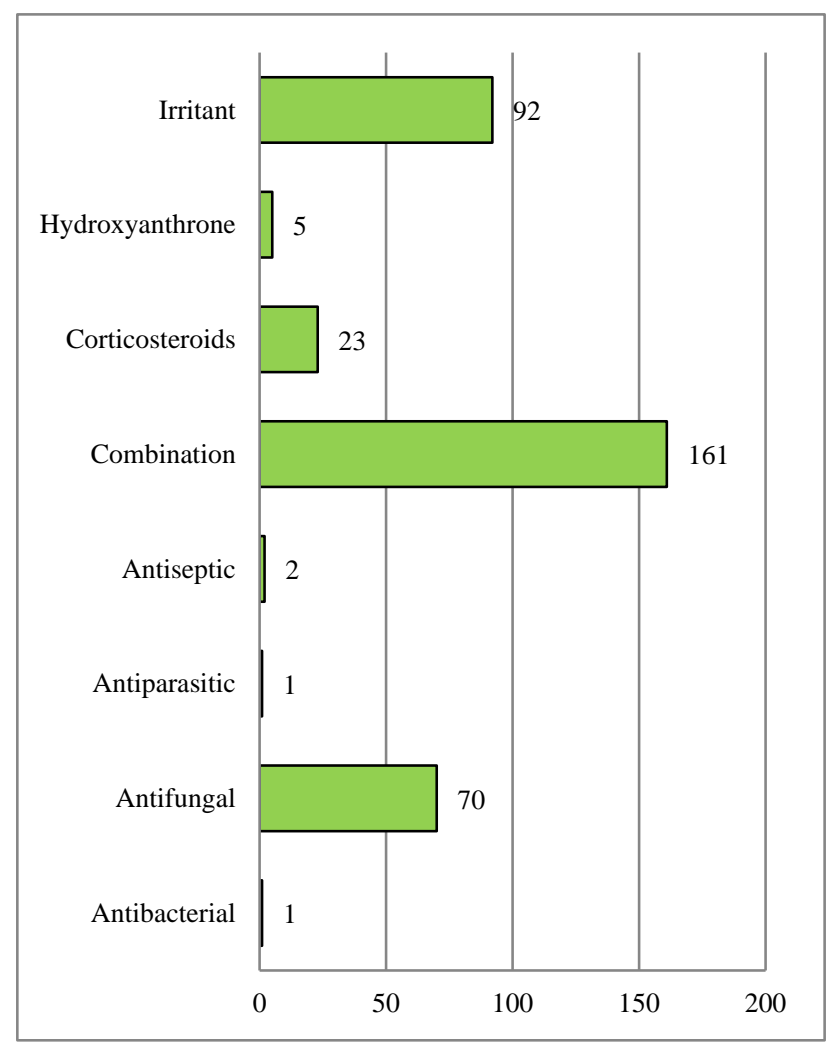

Figure 1: Different types of topical agents categorized on the basis of its nature and active ingredients.

Brand names of commonly misused steroid-based preparations were quadriderm, fourderm, ring guard, dermi 5, betnovate, itchguard, panderm, keto - B and candid B, which could be procured OTC without prescription. Irritant preparations like derobin, zalim, sapat lotion, B-tex, and kharij lotion were infrequently used.
Effects of application of the topical agents as selfmedication to rid from the superficial fungal infection were, $111(52 \%)$ participants got relief for some time when applied and re-occurrence of infection after stopping its use, among 98 (45\%) participants infection was aggravated when topical agents were applied and no effect was seen amongst 6 (3\%) participants (Figure 2).

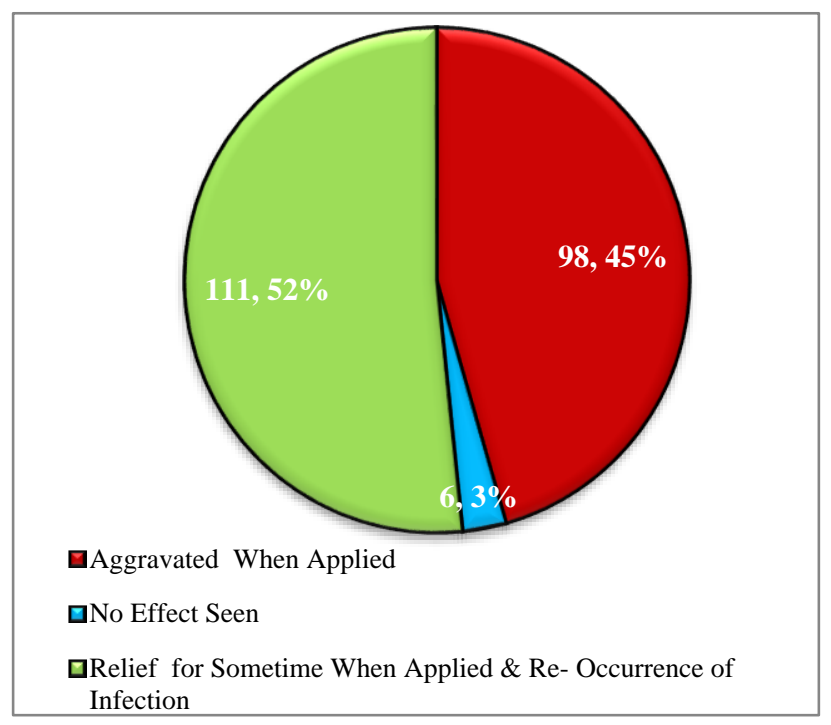

Figure 2: Adverse effects profile of topical agents on the study population.

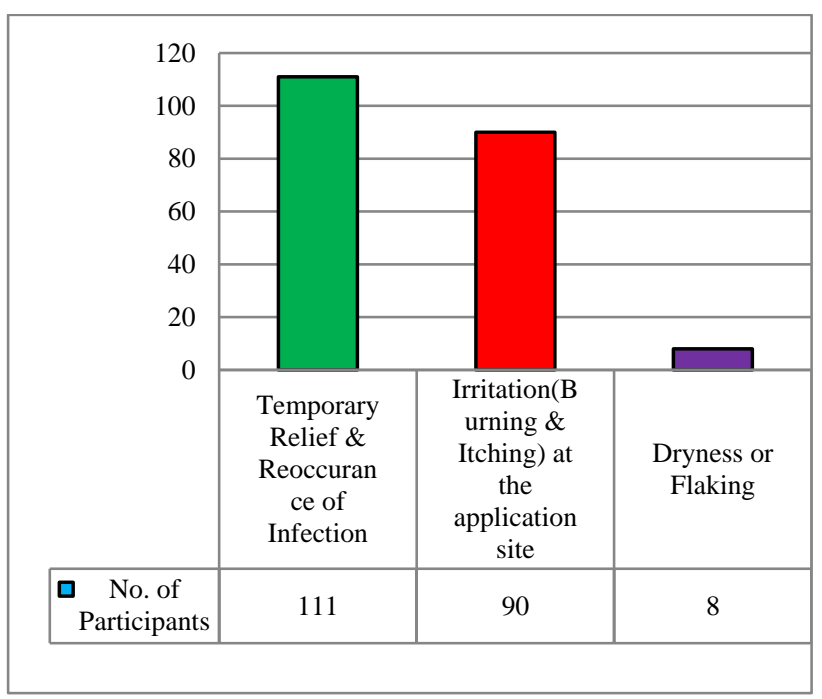

Figure 3: Aggravation of infection produced by topical agents after application.

Among 215 participants, 111 participants got temporary relief from the infection while the infection was reoccurred after stopping the topical agents. 98 participants showed the aggravated effect to topical agents among whom 90 participants showed effects like irritation, burning, itching and redness at the application site, while 8 participants showed dryness or flaking of skin and from these 98 participants, 10 participants showed both irritation and dryness of skin. Where 6 
participants did not show any effect after applying topical agents (Figure 3).

\section{DISCUSSION}

Superficial fungal infections are more common today than ever before and it is widely prevalent in India is rising. Collectively it is the $4^{\text {th }}$ leading cause of nonfatal health burden in 2010 and 2013 globally. ${ }^{13}$ A myriad of atypical presentations due to a complex interplay of agent factors like true resistance, parasitism. The host factors like changing clothing habits, casual health seeking attitude, lack of adherence to standard therapy and social factors like, hesitation to seek medical advice due to involvement of groins, gluteal regions or the inframammary region, unaffordable consultation charges of dermatologist, high cost of branded medication and dermatological products. ${ }^{14,20,21}$

Due to such factors patients gets engaged in using selfmedication. It has become a common healthcare practice among the population. The rise of this trend is because in India, it is effortless and easy to access over the counter medication which also contains several FDC topical agents like steroids, irritants and antifungal which provides quick symptomatic relief due to the antiinflammatory properties of steroids. ${ }^{14}$ As the lack of knowledge about the side effects of such topical agents and availability of such drugs without prescriptions at cheaper rates from variety of sources as the rules regarding dispensing of medication are not so stringent. Due to this reason, many pharmacists bestow medication to patient without prescriptions. It was observed that easy access and injudicious use of such medication has resulted into the aggravation of the infection and it's becoming difficult in treating them. And this hampered patient's quality of life by increasing the suffering in terms of physical, social and psychological. It also increases the financial burden as the most of the skin infections are chronic and requires longer duration of treatment.

Spaced out from steroids, certain other topical agents such as zalim, sapat, B-tex, kharij and ringozone are frequently applied by patients. The commercial advertisements in mass media appealing herbal source and guaranteed efficacy in all kinds of skin rash charm the patients. In addition, there is a misunderstanding among the public that the irritant response provoked by the above-mentioned agents will cure the disease.

Even though the frequent occurrence of the problem, there is a rarity of wide-ranging studies assessing various aspects of topical agents abuse in superficial fungal infection. A recent study by Dutta et al, in 100 cases of tinea incognito reported increasing involvement of face and betamethasone dipropionate as the most commonly abused steroid with a large chunk of the prescription from pharmacists (78\%). ${ }^{15}$ In another study from Delhi, 2174 outpatient department patients were screened for inappropriate use of topical steroids. About $12 \%$ of the patients admitted using of TC and dermatophytosis was the leading cause of steroid abuse (38.4\%). Betamethasone valerate-based medications were most commonly used with friends/relatives as the common source of prescription (33.2\%). ${ }^{16}$ Furthermore, a case series of tinea incognito resulting from misuse of potent TCs has been reported by Sheth et al, Janney et al, in 100 cases revealed tinea cruris as the most common clinical presentation (44\%), whereas $32 \%$ of the study population consulted a dermatologist on developing a rash, the majority $(68 \%)$ used OTC topical medications recommended by pharmacists and friends/relatives, general practitioners. $^{17} \mathrm{~A}$ total of 33 different commercially available OTC preparations were used, were $77.94 \%$ of the patients used steroid based preparations in which $63.23 \%$ of the patients using these OTC preparations reported to have adverse effects. ${ }^{14}$ In another study carried by Mohanty et al, among 200 patients, they found the prevalence of self-medication for dermatological conditions among patients was $62.26 \%$. Most of the drugs for self-medication were topical (creams) FDC of antifungal and steroids. Around 78.12\% of the self-medication information was obtained from the chemists. $^{13}$

Therefore, to define self-medication pattern for superficial fungal infections and assessing all types of OTC topical agent's abuse in dermatology outpatient the present study was designed in contrast to previous studies focusing mainly on steroid abuse. Results will help to promote rational uses of medicines and discourage selfmedication practices. In addition, our study was solely based on patients belong to Mumbai city suffering from dermatophytosis.

In the present study, tinea cruris was the most common clinical presentation $(50.23 \%)$, differing from a recent study which reported tinea cruris to be $44 \%$ and tinea faciei as the predominant type. ${ }^{14,15}$ This difference could be because of the highly humid climatic condition of Mumbai city and the faster running lifestyle of the population using occlusive clothing for prolonged periods.

The casual health seeking attitude of Indian patients is reflected in this study with only $7.44 \%$ of the patients consulting the dermatologist on developing a skin rash. The data shows decrease in the patients approaching dermatologist in the previous studies wherein they were approached by (14-40) \% of patients. ${ }^{18,19}$ These decreasing percentage may have different reasons like high consultation fees of dermatologist, easy access to topical agents wherein patient can easily able to buy them without prescription. The major source of advice for the use of self-medication were pharmacists $(45.58 \%)$ and friends/relatives $(26.97 \%)$ while advertisement $(8.83 \%)$ are also influencing patients in engaging them in selfmedication. Pharmacists have been a major source for obtaining topical agents (20-78) \% in the earlier studies 
also. ${ }^{15,16}$ Hence, educating pharmacists about the adverse effects of irrational use of such topical agents is crucial. While rules for dispensing such medication should be made stringent so that patient would not get easy access to them.

In this study, $92.55 \%$ of patients used 61 different commercially available topical agents, which shows on an average (3.52) medication are been used by patients for self-medication before consulting for rational treatment i.e. almost the doubles the number of medication (33) used to be found in previous studies carried by Janney et al, FDC $(52.09 \%)$ were commonly used, and clobetasol based preparations were leading dissimilar other previous Indian studies wherein betamethasone was the most habitually used OTC preparation. ${ }^{15,16}$ Apart from these, irritants like sapat lotion $(42.79 \%)$ was found to be another major topical agent used by patients in this study which shows decreasing trend of using only steroids (11.16\%) as topical agent. This highlights the increasing demands or use of FDC and irritants in present days.

As every medication by means of injudicious and inappropriate practice has some adverse effects it was also observed in this study were temporary relief and recurrence of infection $(51.62 \%)$ wherein burning sensation and itching $(41.86 \%)$ was the most common adverse effect experienced by the patients, whereas dryness and flaking skin (3.72\%) was observed this study. Burning sensation and flaking skin was more common in patients who used irritants while temporary relief was due to the anti-inflammatory actions of steroids which resulted into the worsening of the existing lesions and making it difficult to treat.

In a crowded city like Mumbai, where literacy rate is $89.73 \%$, it does not come as a surprise that most of the patients $(84.18 \%)$ had neither heard of steroids nor were aware of their adverse effects. ${ }^{14}$ The growing threat of OTC medication misuse in India is evident from this study. In this direction the Indian Association of Dermatologists, Venereologists and Leprologists has already started a nationwide campaign against topical steroid abuse. ${ }^{22}$ Now it is the decisive point in time for drug regulatory agency to keep a check on minimizing the number of FDC and OTC topical preparations and sale solely on valid prescriptions.

\section{CONCLUSION}

Our study confirms that self-medication is widely practiced among patients with a superficial fungal skin infection in Mumbai. From 360 patients screened for this study, 215 patients were found to be using topical agents for self-medication. Patients have used around 61 different brand names of topical agents that belong to the category of irritants, corticosteroids, antifungal and combinations containing corticosteroids, antifungal and antibacterial. After applying these topical agents, the different effects of irritation, burning sensation, itching, dryness or flaking of skin at the application site was observed due to the content and type of topical agents. The pharmacist was the major source for obtaining these topical agents. Other than that, from various sources like friends, relatives, television advertisements and from selfpast experience patients have used those topical agents as self-medication. From the current study, we can conclude that there is a need to counsel and discourage patients from self-medication and regulations for dispensing the prescribed and non-prescribed drugs should be made stringent as it is increasingly posing as a huge health hazard.

\section{Recommendations}

Regulations for dispensing the prescribed and nonprescribed drugs should be made stringent. Strictly avoid any topical agents like irritants, corticosteroid and antifungal preparation consisting steroid. Strong counselling emphasizing the hazards of fixed dose combinations (FDCs) is necessary. Points up the significance of regularity of medication and adherence to the advice of the dermatologist. Avoid tight garments such as jeans, leggings and jeggings. Wear loose, cotton garments. Deject sharing of bed linen if possible, towels and clothes. Regularly washes towels and bed linen. Take a regular shower. Wear clothes only after properly drying the body. Wash infected clothes separately. Use non-occlusive footwear. Counsel and explain all this are time-consuming but, it is crucial to ensure the compliance and to enhance the awareness levels of the patients. One can give pamphlets or use posters to increase adherence to all described measures.

\section{ACKNOWLEDGEMENTS}

Authors would like to thank Department of Infectious Diseases (IDD), KEM Hospital, MUHS, Mumbai and Department of Dermatology, Seth G. S. Medical College and KEM Hospital, Parel, Mumbai.

\section{Funding: No funding sources}

Conflict of interest: None declared

Ethical approval: The study was approved by the Institutional Ethics Committee

\section{REFERENCES}

1. Hoog DS, Monod M, Dawson T, Boekhout T, Mayser P, Graser Y. Skin Fungi from Colonization to Infection. Microbiology Spectrum. 2017;5(4):1-17.

2. Sajith M, Lokhande K, Padma S, Pawar A. Prevalance of various skin disorders and prescribing pattern of antihistamines in tertiary care hospital, Pune. Int J Pharma Sci Res (IJPSR). 2014;5(3):73-77.

3. Joel JJ, Jose N, Shastry CS. Patterns of skin disease and prescribing trends in rural India. Sch Acad J Pharm. 2013;2(4):304-9. 
4. Das K. Pattern of dermatological diseases in Gauhati medical college and hospital Guwahati. Indian $\mathbf{J}$ of Dermatology. 2003;69(1):16-18.

5. Piro DJ. Pharmacotherapy. New York: McGraw Hill. 1st edition: 2008: 1741-1785.

6. Grover S, Ranyal R, Bedi M. A cross section of skin diseases in rural Allahabad. Indian J Dermatology. 2008;53(4):179-81.

7. Yuwnate A, Chandane R, Giri K, Yunati M, Sirsam S. A multicentre pharmacoepidemiological study of dermatological disorders in Wardha district. Int $\mathbf{J}$ Basic Clinical Pharmacology. 2013;2(6):751-56.

8. Sclafer J, Slamet L, Visscher DG. Appropriateness of self-medication: method development and testing in urban Indonesia. J Clinical Pharmacy Therapeutics. 1997;22(4):261-72.

9. Phalke V, Phalke D, Durgawale P. Self-medication practices in rural Maharashtra. Indian J Community Medicine. 2006;31(1):34-5.

10. Sharma R, Verma U, Sharma C, Kapoor B. Selfmedication among urban population of Jammu city. Indian J Pharmacology. 2005;37(1):40-3.

11. Shakoor O, Taylor RB, Behrens RH. Assessment of the incidence of substandard drugs in developing countries. Tropical Medicine Int Health. 1997;2(9):839-45.

12. Elghblawi E. Extensive 'Tinea Incognito' Due to Topical Steroid: A Case Report. JMED Res. 2013;2013(2013):1-3.

13. Deb P, Mohanty I, Slathia I, Verma V. Drug utilisation and self-medication pattern of anti-fungal drugs in dermatology outpatient department of a tertiary care hospital. Int $\mathbf{J}$ Basic Clinical Pharmacology. 2017;6(9):2189-92.

14. Dabas R, Janney MS, Subramaniyan R, Arora S, Lal VS, Donaparthi N. Use of over-the-counter topical medications in dermatophytosis: A cross-sectional, single-center, pilot study from a tertiary care hospital. Indian J Drugs in Dermatology. 2018;4(1):13-17.

15. Dutta B, Rasul ES, Boro B. Clinico-epidemiological study of tinea incognito with microbiological correlation. Indian J Dermatol Venereol Leprol. 2017;83(3):326-31

16. Mahar S, Mahajan K, Agarwal S, Kar HK, Bhattacharya SK. Topical corticosteroid misuse: The scenario in patients attending a tertiary care hospital in New Delhi. J Clinical Diagnostic Res. 2016;10(12):16-20.

17. Sheth HJ, Rathod SP, Chaudhary RG, Malhotra SD, Patel PR. Tineaincognito with unjustified use of potent topical corticosteroids: A case series. Int $\mathbf{J}$ of Basic and Clinical Pharmacology. 2017;6(8):208790.

18. Kim WJ, Kim TW, Mun JH, Song M, Kim HS, Ko H $\mathrm{C}$, et al. Tinea incognito in Korea and its risk factors: Nine-year multicenter survey. J Korean Med Sci. 2013;28(1):145-51.

19. Ansar A, Farshchian M, Nazeri H, Ghiasian SA. Clinico-epidemiological and mycological aspects of tinea incognito in Iran: A 16 years study. Med Mycol J. 2011;52(1):25-32.

20. Dogra S, Uprety S. The menace of chronic and recurrent dermatophytosis in India: Is the problem deeper than we perceive. Indian Dermatol Online J. 2016;7(2):73-6.

21. Narang T, Mahajan R, Dogra S. Dermatophytosis: Fighting the challenge: Conference proceedings and learning points. September 2-3, 2017, PGIMER, Chandigarh, India. Indian Dermatol Online J. 2017;8(6):527-33.

22. Kumar S, Goyal A, Gupta YK. Abuse of topical corticosteroids in India: Concerns and the way forward. J Pharmacol Pharmacother. 2016;7(1):1-5.

Cite this article as: Mahajan SA, Deshmukh SS, Rawal JR. Observational cross-sectional study to evaluate the effects of self-medication with topical agents used by patients for superficial fungal skin infection at tertiary care hospital in Mumbai. Int $\mathbf{J}$ Basic Clin Pharmacol 2020;9:796-801. 